\title{
Race and smoking status associated with paclitaxel drug response in patient-derived lymphoblastoid cell lines
}

\author{
Farida S. Akhtari ${ }^{\mathrm{a}, \mathrm{b}}$, Tammy M. Havener ${ }^{\mathrm{c}}$, Daniel L. Hertz, ${ }^{\mathrm{d}}$ \\ Jeremy Ash ${ }^{\mathrm{b}}$, Alexandra Larson ${ }^{\mathrm{b}}$, Lisa A. Carey, ${ }^{\mathrm{e}}$ \\ Howard L. McLeod ${ }^{f, g}$ and Alison A. Motsinger-Reif ${ }^{g, h}$
}

The use of ex-vivo model systems to provide a level of forecasting for in-vivo characteristics remains an important need for cancer therapeutics. The use of lymphoblastoid cell lines (LCLs) is an attractive approach for pharmacogenomics and toxicogenomics, due to their scalability, efficiency, and cost-effectiveness. There is little data on the impact of demographic or clinical covariates on LCL response to chemotherapy. Paclitaxel sensitivity was determined in LCLs from 93 breast cancer patients from the University of North Carolina Lineberger Comprehensive Cancer Center Breast Cancer Database to test for potential associations and/or confounders in paclitaxel dose-response assays. Measures of paclitaxel cell viability were associated with patient data included treatment regimens, cancer status, demographic and environmental variables, and clinical outcomes. We used multivariate analysis of variance to identify the in-vivo variables associated with ex-vivo dose-response. In this unique dataset that includes both in-vivo and ex-vivo data from breast cancer patients, race $(P=0.0049)$ and smoking status $(P=0.0050)$ were found to be significantly associated with ex-vivo dose-response in LCLs. Racial differences in clinical dose-response have been previously described, but the smoking association has not been reported. Our results indicate that in-vivo

\section{Introduction}

An important challenge in the development of anticancer therapies is the paucity of robust model systems for precise translation from the preclinical to clinical setting. One approach uses human B cell lymphocytes from peripheral blood that are infected ex vivo with the Epstein Barr virus (EBV) to create immortalized human lymphoblastoid cell lines (LCLs) [1]. LCLs are a widely used model system in several fields, including immunology, toxicogenomics, and pharmacogenomics [2]. LCLs have been successfully used in genetic and functional studies to find associations with genetic variations and to investigate the response of numerous chemotherapeutics, offering insights into the mechanism of action or resistance [3-5]. Several such

Supplemental Digital Content is available for this article. Direct URL citations appear in the printed text and are provided in the HTML and PDF versions of this article on the journal's website, www.pharmacogeneticsandgenomics.com. smoking status can influence ex-vivo dose-response in LCLs, and more precise measures of covariates may allow for more precise forecasting of clinical effect. In addition, understanding the mechanism by which exposure to smoking in-vivo effects ex-vivo dose-response in LCLs may open up new avenues in the quest for better therapeutic prediction. Pharmacogenetics and Genomics 31: 48-52 Copyright (C) 2021 Wolters Kluwer Health, Inc. All rights reserved.

Pharmacogenetics and Genomics 2021, 31:48-52

Keywords: dose-response, lymphoblastoid cell lines, paclitaxel, patient-derived cell lines, pharmacogenomics, smoking

aDepartment of Biological Sciences, ${ }^{b}$ Bioinformatics Research Center, North Carolina State University, Raleigh, 'Pharmacotherapy and Experimental Therapeutics, University of North Carolina at Chapel Hill, Chapel Hill, North Carolina, ${ }^{d}$ Department of Clinical Pharmacy, University of Michigan College of Pharmacy, Ann Arbor, Michigan, ${ }^{e}$ Division of Hematology/Oncology, University of North Carolina, Chapel Hill, North Carolina, ' 'University of South Florida Taneja College of Pharmacy, Tampa, Florida, ${ }^{9}$ Center for Pharmacogenomics and Individualized Therapy, University of North Carolina at Chapel Hill, Chapel Hill and hiostatistics and Computational Biology Branch, National Institute of Environmental Health Sciences, Durham, North Carolina, USA

Correspondence to Alison Motsinger-Reif, $\mathrm{PhD}$, Biostatistics and Computational Biology Branch, National Institute of Environmental Health Sciences, 111 TW Alexander Drive, Durham, NC 27709, USA

Tel: +984 287 3705; e-mail: alison.motsinger-reif@nih.gov

Received 2 March 2020 Accepted 22 June 2020

discoveries using LCLs have led to successful clinical translations $[6,7]$.

The model is highly scalable, efficient, and cost-effective with fewer known confounding issues compared with other preclinical models [3]. LCLs, such as those in the 1000 Genomes Project [8], are created from individuals from a wide range of demographic populations and exposures. As with any model system, its success will be dependent on properly understanding confounders. Research has demonstrated that growth rate, batch effects and the sex, ethnicity, and race of the samples can influence dose-response in LCLs [9-12]. These known confounders can be controlled for by experimental design and execution, quality control, and appropriate control of covariates in the statistical analyses [3]. However, to the best of our knowledge, the effect of other in-vivo factors, such as in-vivo exposures, on ex-vivo dose-response in LCLs has not been studied. 
Here, we used patient-derived LCLs from breast cancer patients to examine the effect of a number of demographic and clinical in-vivo variables on ex-vivo paclitaxel dose-response.

\section{Materials and methods}

The patient cohort consisted of 93 breast cancer patients from a prospective trial of genetic heterogeneity (LCCC 9830), the University of North Carolina (UNC) Lineberger Comprehensive Cancer Center (LCCC) that included LCLs created from pretreatment blood drawn from newly diagnosed breast cancer patients. These 93 were selected from the overall study cohort because of receiving paclitaxel as part of their systemic therapy, as described in detail in Hertz et al. [13]. The in-vivo patient data included treatment regimens, cancer status, demographic and environmental variables, and prospectively ascertained clinical outcomes for each patient. Informed consent was obtained from all participants. This study adhered to the 1964 Declaration of Helsinki and its later amendments and the protocol was approved by the UNC Institutional Review Board.

In brief, a $30-\mathrm{mL}$ blood sample was collected from each patient pretreatment, at the time of enrollment. Lymphocytes from blood samples were infected with EBV to create immortalized human LCLs, as previously described [14]. The LCLs were then grown on 384 well plates, and percent viability for each cell line was measured at each of 10 concentrations (nM) of paclitaxel (100, $250,350,450,500,550,600,700,900$, and 1200). Details of these methods are found in the Supplemental Material, Supplement digital content 1, http://links.lww.com/FPC/ B381.

A QC pipeline, described in detail in Brown et al., was applied prior to statistical analysis $[4,15,16]$. To avoid issues with imbalanced classes and increase statistical power for multivariate analysis of variance (MANOVA), observations whose categorical level had a frequency $\leq 10$ were filtered out, and highly correlated responses were removed (Supplemental Methods, Supplemental Figs. S1 and S2, Supplement digital content 1, http://links. lww.com/FPC/B381 and Supplemental Tables S1 and S2, Supplement digital content 1, http://links.lww.com/FPC/ B381). Multivariate analyses using the complete dose-response profile have more power and maybe more robust than univariate summary measures, such as the $A_{50}$ [16]. Hence, to identify in-vivo variables associated with ex-vivo dose-response in LCLs, we conducted a one-way MANOVA using four minimally correlated dose-response variables as the response and each categorical in-vivo variable of interest as predictor variables. When the in-vivo variables were ordinal or continuous, multivariate response regression was used. We used the BenjaminiHochberg method [17] with a false discovery rate of $q<0.10$ to ascribe significance. Additional supporting methods and analyses of the $\mathrm{AC}_{50}$ response, a two-way MANOVA with race and smoking status, and comparisons of ex-vivo response to in-vivo clinical outcomes are described in Supplemental Material, Supplement digital content 1, http://links.lww.com/FPC/B381. Analyses were conducted using R [18].

\section{Results}

Results from the one-way MANOVA analyses are shown in Table 1. Race and smoking status in vivo were found to be independently, significantly associated with ex-vivo dose-response with moderate effect size. Table 2 shows estimated contrasts for smoking status and their significance after a Bonferroni correction. We observe that individuals who never smoked and former smokers were less sensitive to paclitaxel ex vivo than current smokers at higher concentrations. However, there is no significant difference between never and former smokers. This is also shown in Fig. 1a, which illustrates that current smokers have lower cell viability than former smokers and never smokers, at $600 \mathrm{nM}$ of paclitaxel. Analyses evaluating racial differences in response followed similarly, and results are shown in Fig. 1b and Table 3. Figure 1b

Table 1 Results from one-way multivariate analysis of variance with the selected four dose-response variables as the response and each in-vivo variable of interest as predictor variables

\begin{tabular}{llcc}
\hline & \multicolumn{1}{c}{ Variable } & $P$ value & Corrected $P$ value \\
\hline 1 & Race & 0.0049 & 0.0501 \\
2 & Smoke & 0.0050 & 0.0501 \\
3 & Neuropathy & 0.2966 & 0.9359 \\
4 & ER & 0.3771 & 0.9359 \\
5 & Regimen & 0.4029 & 0.9359 \\
6 & ER or PR & 0.4621 & 0.9359 \\
7 & Total weeks & 0.5052 & 0.9359 \\
8 & Menopause & 0.5108 & 0.9359 \\
9 & Age & 0.5187 & 0.9359 \\
10 & Grade & 0.5515 & 0.9359 \\
11 & Myalgia & 0.6231 & 0.9359 \\
12 & StagePre & 0.6687 & 0.9359 \\
13 & Neutropenia & 0.7683 & 0.9359 \\
14 & Follow-up status & 0.7696 & 0.9359 \\
15 & NumCycles & 0.7958 & 0.9359 \\
16 & Dose interval & 0.8109 & 0.9359 \\
17 & StageFinal & 0.8366 & 0.9359 \\
18 & Response nontaxane & 0.8423 & 0.9359 \\
19 & Response taxane & 0.9708 & 0.9715 \\
20 & HER2 & 0.9715 & 0.9715 \\
& & & \\
\hline
\end{tabular}

The Benjamini-Hochberg method was used to control for multiple testing correction with a false discovery rate of $q<0.10$. Race and smoking status were found to be significantly associated with ex-vivo dose-response in LCLs after multiple testing correction.

Age, age at diagnosis (in years); dose interval, paclitaxel dose interval; ER, estrogen receptor status (positive or negative); ER or PR, estrogen or progesterone receptor-positive (yes or no); follow-up status, follow-up status; grade, grade of tumor at diagnosis; HER2, human epidermal growth factor receptor 2 status (positive or negative); menopause, menopause status at diagnosis (pre or post); myalgia, myalgia status (yes or no); neuropathy, grade $3+$ neuropathy status (yes or no); neutropenia, neutropenia status (yes or no); NumCycles, number of paclitaxel chemotherapy cycles; race, self-reported race; Regimen, paclitaxel regimen (first or second); response nontaxane, response to nonpaclitaxel therapy; response taxane, response to paclitaxel therapy; smoke, smoking status (current, former or never smoker); StageFinal, final cancer stage; StagePre, cancer stage before start of treatment; total weeks, total weeks of treatment; LCLs, lymphoblastoid cell lines; MANOVA, multivariate analysis of variance 
Table 2 Estimated pairwise contrasts and their $P$ values for the multivariate dose-response profile and smoking status

\begin{tabular}{|c|c|c|c|c|c|c|}
\hline Contrast & $1200 \mathrm{nM}$ & $600 \mathrm{nM}$ & $500 \mathrm{nM}$ & $100 \mathrm{nM}$ & $P$ value & Bonferroni corrected $P$ value \\
\hline Former smoker-never smoked & 0.17 & 4.15 & 3.53 & 1.90 & 0.0720 & 0.2160 \\
\hline Former smoker-current smoker & 3.50 & 13.78 & 0.23 & 0.41 & 0.0141 & $0.0424^{*}$ \\
\hline Never smoked-current smoker & 3.33 & 9.62 & -3.30 & -1.48 & 0.0135 & $0.0404^{\star}$ \\
\hline
\end{tabular}

The multivariate dose-response analysis used four minimally correlated responses at 100, 500,600, and $1200 \mathrm{nM}$ of paclitaxel out of the ten measured responses (see Supplemental Methods, Supplement digital content 1, http://links./ww.com/FPC/B381 for details).

Number of individuals in each category: current smoker $=12$, former smoker $=27$, never smoker $=54$.

shows that at $600 \mathrm{nM}$ of paclitaxel, lower cell viability is observed in individuals who are black compared with individuals who are white. The entire dose-response profile for all individuals is shown in Supplemental Fig. S5, Supplement digital content 1, http://links.lww.com/FPC/ B381 segregated by (a) smoking status and (b) race.

\section{Discussion}

It is well established that LCL growth rate, batch effects, and the sex and race of the individuals affect the dose-response phenotype in LCLs [9-12]. However, to the best of our knowledge, the effect of other in-vivo factors on ex-vivo dose-response in LCLs has not been studied. Our results show an expected and confirmatory effect of race [13] and an unexpected and surprising effect of an in-vivo environmental factor, smoking status, on ex-vivo dose-response in LCLs. Interestingly, the effect size of smoking status (partial $\eta^{2}=0.096$ ) is on the same order of magnitude as that of the race (partial $\eta^{2}=0.13$ ) (Supplemental Table S3, Supplement digital content 1, http://links.lww.com/FPC/B381), indicating that the influence of smoking status on dose-response is comparable to that of racial ancestry.

While smoking status was not significantly associated with the clinical outcome in our study, it is known that smoking can impact patient outcomes. Studies have shown that smoking can worsen patients' prognoses and survival outcomes after anticancer therapy [19,20]. Studies have also shown resistance to chemotherapeutics in cancer cells exposed to nicotine [21,22]. However, our results suggest that LCLs from active smokers were more sensitive ex vivo to paclitaxel, at higher concentrations. This potential discordance between the clinical observation and laboratory sensitivity patterns needs to be better understood as the use of ex-vivo model systems expands.

Whether any such association is considered a confounder or a covariate is unclear, and could be contextual on the basis of study design, but our results demonstrate for the first time that smoking status of LCL donors is linked to ex-vivo response. Unfortunately, for many of the publicly available LCL results, this data is not available. Our results suggest that smoking status of donors from whom LCLs are cultured should be recorded and considered in analysis if possible. Although factors, such as batch effect, that are clearly only associated with ex-vivo response need to clearly be adjusted for, other factors that may be true indicators of clinical in-vivo response should be modeled in a hypothesis-specific fashion.

This begs the question of how in-vivo smoking affects ex-vivo dose-response because the LCLs are no longer exposed to the environmental factor. We hypothesize that the effect of smoking in vivo could persist in the LCLs through epigenetic mechanisms, such as DNA or histone methylation. One such pharmacoepigenetic study by Zhang and Zhang identified epigenetic variations associated with clofarabine-induced cytotoxicity in LCLs [23]. Recent studies of the acquired environmental genetic change clonal hematopoiesis of indeterminant potential suggest that these acquired genetic changes can influence neutropenia risk after chemotherapy for solid tumors [24]. Functional experiments using chromatin immunoprecipitation assays and RNA-sequencing followed by differential gene expression analyses would be required to investigate this hypothesis and understand the mechanism by which exposure to smoking in-vivo effects ex-vivo dose-response.

Although our study has identified an impactful result of the influence of an in-vivo environmental exposure on ex-vivo drug response in LCLs, these results need to be interpreted in context of several limitations. We were unable to detect strong associations between in-vivo clinical outcomes and ex-vivo drug response due to limited sample size and power, in addition to other general limitations to the model, which have been previously discussed in detail [3,7]. Notably, the LCLs used in our study were created from lymphocytes of breast cancer patients, who all received multifaceted treatment strategies, including combination chemotherapy and anti-HER2 treatment, when indicated $[3,7]$. The taxane response outcome on the basis of tumor size was collected prior to and after each line of treatment, such that the taxane response would be independent of other chemotherapy (anthracycline/cyclophosphamide) but would include concurrent HER2 targeted treatment ( 12 weeks). The LCL model cannot capture the complex interplay of multiple treatment strategies with clinical outcomes. Additionally, smoking status was patient-reported and reflects a categorical simplification of what is likely a continuum of biological risk. 

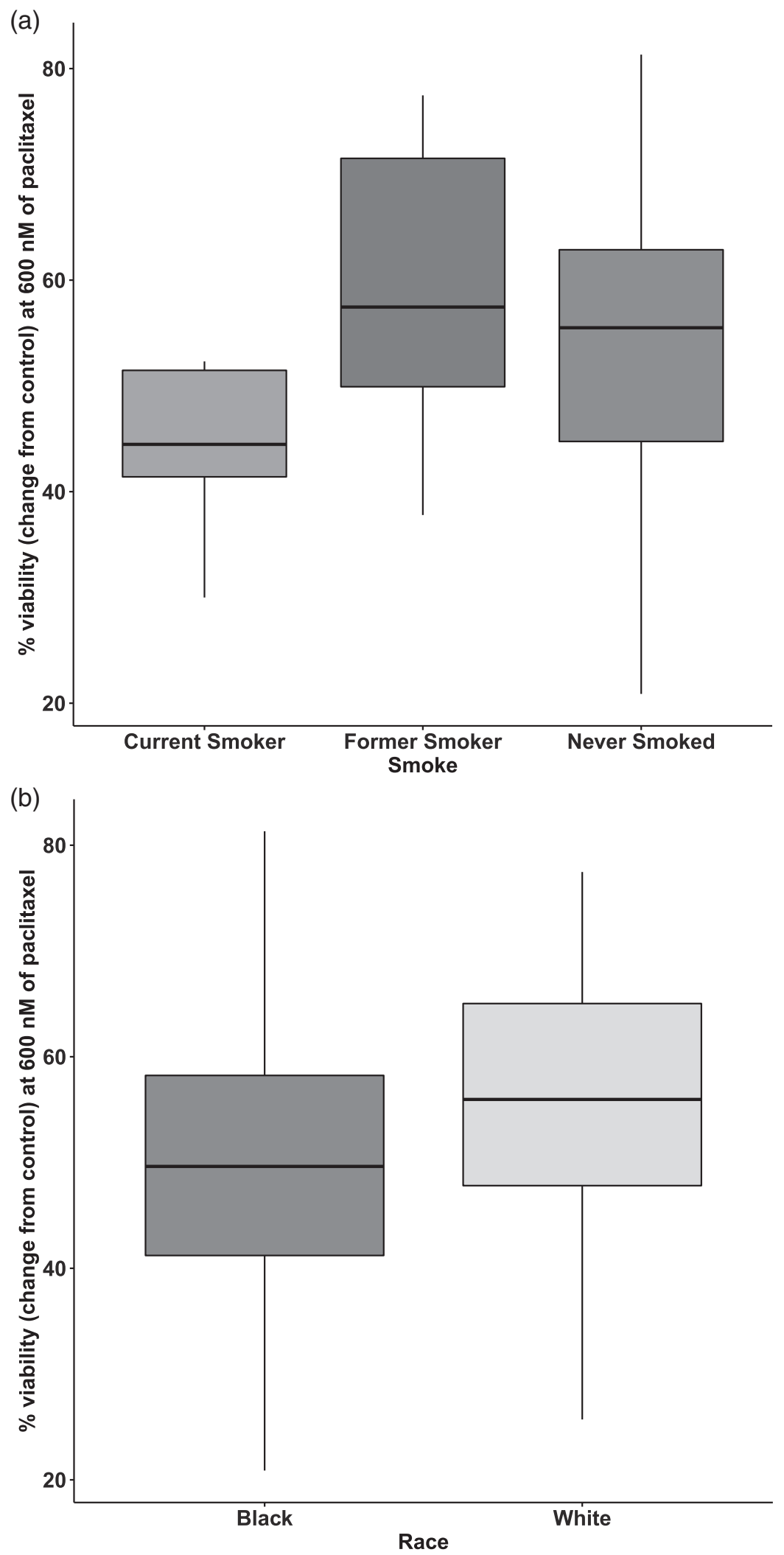

Boxplots showing paclitaxel response stratified by (a) smoking status and (b) race. Percent viability (change from control) at $600 \mathrm{nM}$, as an example concentration of paclitaxel, in the patient-derived cell lines stratified by (a) smoking status and (b) race. (a) Current smokers have significantly lower cell viability compared with the other smoking categories. The difference in cell viability between former smokers and never smokers was not found to be significant. Number of individuals in each category: current smoker $=12$, former smoker=27, never smoker=54. (b) Significantly lower cell viability is observed in individuals who are black compared with those who are white. Number of individuals in each category: black=24, white $=64$. 
Table 3 Estimated pairwise contrasts and their $P$ values for the multivariate dose-response profile and race

\begin{tabular}{lccccc}
\hline Contrast & $1200 \mathrm{nM}$ & $600 \mathrm{nM}$ & $500 \mathrm{nM}$ & $100 \mathrm{nM}$ & $P$ value \\
\hline White-black & -1.35 & 5.64 & -0.58 & 0.91 & $0.0049^{* *}$ \\
\hline
\end{tabular}

The multivariate dose-response analysis used four minimally correlated responses at 100,500,600, and $1200 \mathrm{nM}$ of paclitaxel out of the ten measured responses (see Supplemental Methods, Supplement digital content 1, http://links./ww.com/FPC/B381 for details).

Number of individuals in each category: black $=24$, white $=64$.

\section{Conclusion}

This study shows that in-vivo smoking status is significantly associated with ex-vivo dose-response in the LCLs, with a moderate effect size. These results identify an in-vivo environmental exposure, smoking, that is an important factor for LCL dose-response assays and, hence, should be recorded and considered in dose-response analyses when possible. The findings from this study identify an influential, but as of yet unstudied covariate that significantly affects individual variation in dose-response in LCLs.

\section{Acknowledgements}

This study was partially supported by intramural funds from the National Institute of Environmental Health Sciences. It was also supported by R01 CA161608 from the National Cancer Institute. The 9830 study was funded by the Breast Cancer Research Foundation (L.A.C.) and the UNC Breast Cancer SPORE (CA58223).

\section{Conflicts of interest}

There are no conflicts of interest.

\section{References}

1 Scheinfeldt LB, Hodges K, Pevsner J, Berlin D, Turan N, Gerry NP. Genetic and genomic stability across lymphoblastoid cell line expansions. BMC Res Notes 2018; 11:558.

2 National Center for Biotechnology Information (NCBI), U.S. National Library of Medicine (NLM), National Institutes of Health (NIH). https://pubmed. ncbi.nlm.nih.gov/ 1996. [Accessed 2 December 2019].

3 Jack J, Rotroff D, Motsinger-Reif A. Lymphoblastoid cell lines models of drug response: successes and lessons from this pharmacogenomic model. Curr Mol Med 2014; 14:833-840.

4 Brown CC, Havener TM, Medina MW, Jack JR, Krauss RM, McLeod $\mathrm{HL}$, Motsinger-Reif AA. Genome-wide association and pharmacological profiling of 29 anticancer agents using lymphoblastoid cell lines. Pharmacogenomics 2014; 15:137-146.

5 Welsh M, Mangravite L, Medina MW, Tantisira K, Zhang W, Huang RS, et al. Pharmacogenomic discovery using cell-based models. Pharmacol Rev $2009 ; 61: 413-429$.

6 Wheeler HE, Maitland ML, Dolan ME, Cox NJ, Ratain MJ. Cancer pharmacogenomics: strategies and challenges. Nat Rev Genet 2013; 14:23-34.

7 Wheeler HE, Dolan ME. Lymphoblastoid cell lines in pharmacogenomic discovery and clinical translation. Pharmacogenomics 2012; 13:55-70.

8 The 1000 Genomes Project Consortium. A global reference for human genetic variation. Nature $2015 ; \mathbf{5 2 6 : 6 8 - 7 4}$.
9 Choy E, Yelensky R, Bonakdar S, Plenge RM, Saxena R, De Jager PL, et al. Genetic analysis of human traits in vitro: drug response and gene expression in lymphoblastoid cell lines. Plos Genet 2008; 4:e1000287.

10 Ortega VE, Meyers DA. Pharmacogenetics: implications of race and ethnicity on defining genetic profiles for personalized medicine. J Allergy Clin Immunol 2014; 133:16-26.

11 Jack J, Havener TM, McLeod HL, Motsinger-Reif AA, Foster M. Evaluating the role of admixture in cancer therapy via in vitro drug response and multivariate genome-wide associations. Pharmacogenomics 2015; 16:1451-1463.

12 Abdo N, Xia M, Brown CC, Kosyk O, Huang R, Sakamuru S, et al. Population-based in vitro hazard and concentration-response assessment of chemicals: the 1000 genomes high-throughput screening study. Environ Health Perspect 2015; 123:458-466.

13 Hertz DL, Roy S, Motsinger-Reif AA, Drobish A, Clark LS, McLeod HL, et al. CYP2C8*3 increases risk of neuropathy in breast cancer patients treated with paclitaxel. Ann Oncol 2013; 24:1472-1478.

14 Kaufmann WK, Filatov L, Oglesbee SE, Simpson DA, Lotano MA, McKeen $\mathrm{HD}$, et al. Radiation clastogenesis and cell cycle checkpoint function as functional markers of breast cancer risk. Carcinogenesis 2006; 27:2519-2527.

15 Brown C, Havener TM, Everitt L, McLeod H, Motsinger-Reif AA. A comparison of association methods for cytotoxicity mapping in pharmacogenomics. Front Genet 2011; 2:86.

16 Brown CC, Havener TM, Medina MW, Krauss RM, McLeod HL, Motsinger-Reif AA. Multivariate methods and software for association mapping in dose-response genome-wide association studies. Biodata Min 2012; 5:21.

17 Benjamini Y, Hochberg Y. Controlling the false discovery rate: a practical and powerful approach to multiple testing. J R Stat Soc B 1995; 57:289-300.

18 R Core Team (2020). R: A Language and Environment for Statistical Computing, R Foundation for Statistical Computing, Vienna, Austria. https://www.R-project.org/.

19 Gannon NP, King DM, Bedi M. Smoking is predictive of poorer distant metastasis-free and progression free-survival in soft tissue sarcoma patients treated with pre-operative radiotherapy or chemoradiotherapy. Clin Sarcoma Res 2018; 8:7.

20 Fox JL, Rosenzweig KE, Ostroff JS. The effect of smoking status on survival following radiation therapy for non-small cell lung cancer. Lung Cancer 2004; 44:287-293.

21 Dasgupta P, Kinkade R, Joshi B, Decook C, Haura E, Chellappan $\mathrm{S}$. Nicotine inhibits apoptosis induced by chemotherapeutic drugs by up-regulating XIAP and survivin. Proc Natl Acad Sci USA 2006; 103:6332-6337.

22 Türker Şener L, Güven C, Şener A, Adin Çinar S, Solakoğlu S, Albeniz I. Nicotine reduces effectiveness of doxorubicin chemotherapy and promotes CD $44^{+} \mathrm{CD} 24^{-}$cancer stem cells in MCF-7 cell populations. Exp Ther Med 2018; 16:21-28.

23 Zhang X, Zhang W. Pharmacoepigenetics explores the epigenetic contribution to anti-cancer agent-induced cytotoxicities. Stem Cell Epigenetics 2015; 1 :e359.

24 Bolton KL, Gillis NK, Coombs CC, Takahashi K, Zehir A, Bejar R, et al. Managing clonal hematopoiesis in patients with solid tumors. J Clin Oncol 2019; 37:7-11. 